Paper presented at the Particle Accelerator Conference, Portland, OR, May 12-16, 2003.

BNL-71403-2003-CP

\title{
FINAL TEST RESULTS FOR THE SNS RING DIPOLES*
}

\author{
P. Wanderer ${ }^{\dagger}$, J. Jackson, A. Jain, Y.-Y. Lee, W. Meng, Y. Papaphilippou\#, C. Spataro, \\ S. Tepikian, N. Tsoupas, J. Wei, BNL, Upton, NY 11973-5000, USA
}

\section{Abstract}

To meet the performance parameters of the Spallation Neutron Source (SNS) for high beam intensity with low losses, the compact accumulation ring will contain 32 sector dipoles with $1.44 \mathrm{~m}$ effective length and a large aperture, $170 \mathrm{~mm}$. The magnets are built from potted coils and machined pieces of solid iron. When first assembled, the dipoles met the requirements for field uniformity but the rms variation of the integral transfer function (ITF) was much larger than design at both fields of interest, $1.11 \mathrm{~T} \cdot \mathrm{m}$ and $1.33 \mathrm{~T} \cdot \mathrm{m}$, corresponding to proton energies of $1.0 \mathrm{GeV}$ and $1.3 \mathrm{GeV}$ respectively. Based on initial measurements, shims have been added to the return legs or poles, as appropriate, in order to bring the rms variation of the $1.0 \mathrm{GeV}$ ITF to the specification, $0.01 \%$. The value of the ITF rms variation at $1.3 \mathrm{GeV}$ for the shimmed magnets is $0.033 \%$. Sorting the magnets has significantly reduced the load on the correctors due to this ITF variation.

\section{INTRODUCTION}

The Spallation Neutron Source (SNS) is under construction at Oak Ridge National Laboratory [1]. The SNS accumulator ring [2] will contain 32 sector dipoles. To accommodate the high intensity proton beam, the magnet aperture is large. The dipoles are being characterized over the energy range 0.8 to $1.3 \mathrm{GeV}$ to allow for operation over a range of injection energies. The magnets were fabricated at Brookhaven National Laboratory (BNL), using coils and machined iron parts purchased from industry. The yokes are machined from solid 1006 steel. Other parameters of the magnets are given in Table 1. A drawing of the magnet is shown in Fig. 1. This paper reports magnetic measurement results from all 32 dipoles. Measurements of the first 16 dipoles have been published previously [3].

Table 1: Magnet Parameters

\begin{tabular}{|l|l|}
\hline Effective length, $\mathrm{m}$ & 1.44 \\
\hline Gap, $\mathrm{mm}$ & 170 \\
\hline Pole width, mm & 450 \\
\hline$B_{0}$ at $1.0 \mathrm{GeV}(1.3 \mathrm{GeV}), \mathrm{T}$ & $0.77(0.925)$ \\
\hline$I_{\mathrm{op}}$ at $1.0 \mathrm{GeV}(1.3 \mathrm{GeV}), \mathrm{A}$ & $4398(5408)$ \\
\hline SB.dl at $1.0 \mathrm{GeV}(1.3 \mathrm{GeV}), \mathrm{T} \cdot \mathrm{m}$ & $1.11(1.33)$ \\
\hline
\end{tabular}

* SNS is managed by UT-Batelle, LLC, under contract DE-AC0500OR22725 for the U.S. Department of Energy. SNS is a partnership of six U.S. national laboratories: Argonne, Brookhaven, Jefferson, Lawrence Berkeley, Los Alamos, and Oak Ridge.

$\uparrow$ wanderer@ibnl.gov

\# now at ESRF, Grenoble, France

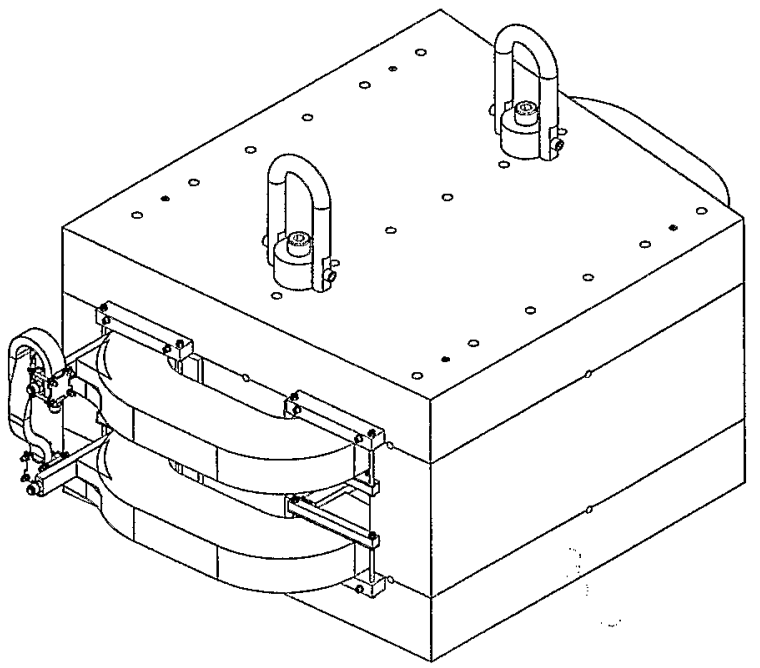

Figure 1: SNS Ring Dipole

\section{FIELD QUALITY REQUIREMENTS}

For the individual magnets, there are field quality requirements for the integral field and for the region near the axial center of the magnet, where the field is twodimensional. (The requirement applies to the integral field after the linear variation of the integral dipole field with horizontal position due to the sector ends is subtracted.) In both cases, the requirements call for $0.01 \%$ uniformity across an aperture of $\pm 100 \mathrm{~mm}$ at the horizontal midplane. Field uniformity near the axial center of the magnet was achieved by locating steel strips $5.19 \mathrm{~mm}$ high and $40 \mathrm{~mm}$ wide at the edges of the poles. Uniformity of the integral field was achieved by adjusting the amount of steel at the corners of a yoke of the prototype dipole in an iterative process that utilized both three-dimensional calculations and measurement.

For the group of magnets, the rms variation of the integral field is to be less than $0.01 \%$. After measurement, the magnets are disassembled in order to install the vacuum chamber, so magnet assembly must be reproducible to $0.01 \%$ in order to assure that the field measurements correctly represent the installed magnets. The six yoke sections (poles, top and bottom plates, side plates) are pinned and bolted to obtain this reproducibility.

\section{MAGNETIC FIELD MEASUREMENTS}

The integral fields in production dipoles are measured with a rotating coil $2.49 \mathrm{~m}$ long and $163.8 \mathrm{~mm}$ in diameter. Tangential sense windings are mounted on the coil [4]. At 1.0 and $1.3 \mathrm{GeV}$, measurements are made at 
the center of the magnet aperture and at positions 50.8 and $101.6 \mathrm{~mm}$ on both sides of center. The regions covered by the coil at these positions overlap, providing considerable redundancy in the data. For a sector magnet, accurate horizontal placement of the measuring coil is essential. (A horizontal displacement of $0.76 \mathrm{~mm}$ changes the integral field by $0.01 \%$.) This is accomplished by a precision magnet stand and a computer controlled coil translation stage. In addition, optical survey is used to locate fiducials on the coil with respect to the magnet fiducials. The survey data are then used to correct the magnetic measurements for any errors in the coil position. The short-term variation (i.e., noise) of the integral measurements is $\sim 0.002 \%$. The reproducibility of the measurements is $\sim 0.01 \%$ when the magnets are removed from and reinstalled on the test stand. The absolute accuracy of the integral field measurement is estimated to be $\sim 0.03 \%$.

To obtain reproducibility of the hysteresis, the magnets were ramped to $4834 \mathrm{~A}(5408 \mathrm{~A})$ for $1.0 \mathrm{GeV}(1.3 \mathrm{GeV})$ operation. Following this cycle, the magnets were ramped up to the same high current. Measurements were then made on the down-ramp.

The as-built dipoles did not meet the specification of a $0.01 \%$ standard deviation in the integral transfer function (ITF) due to variations in the pole gap, as well as apparent variations in the iron properties. The dipoles were shimmed to meet the specifications. A target mean value was established based on the measurements of a few early dipoles. For all the subsequent dipoles, a preliminary measurement was carried out at $1.0 \mathrm{GeV}$ excitation and a shim was installed, if necessary, either in the return leg position (to reduce the ITF) or under the upper pole (to increase the ITF). Most magnets were shimmed to within $\pm 0.02 \%$ of the target value on the first attempt. A few magnets remained outside this margin (which corresponds to the shim resolution of $25 \mu \mathrm{m}$ ) after the first shimming. These magnets were reshimmed.

Initially, the shim thickness was determined based on

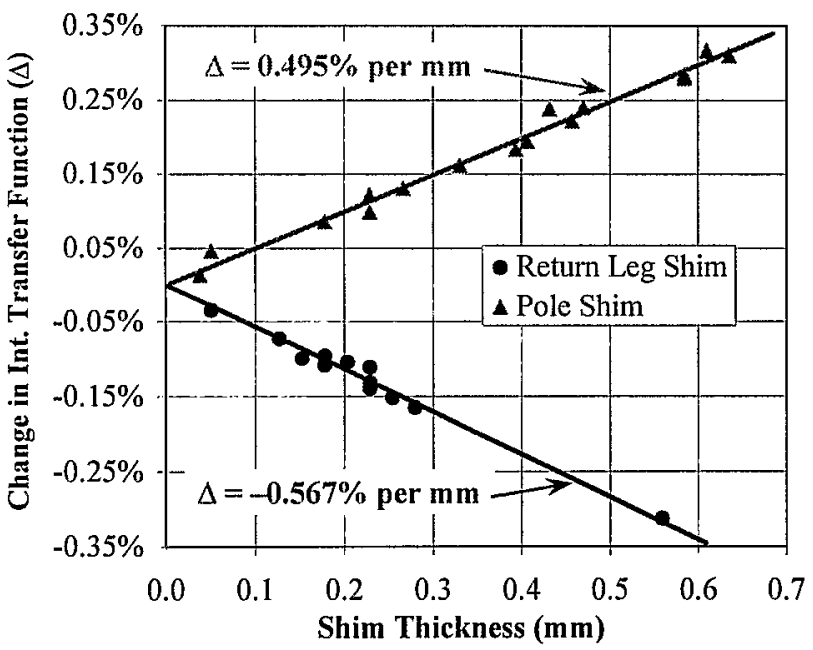

Figure 2: Change in Integral Transfer Function as a function of shim thickness in the pole and the return leg.

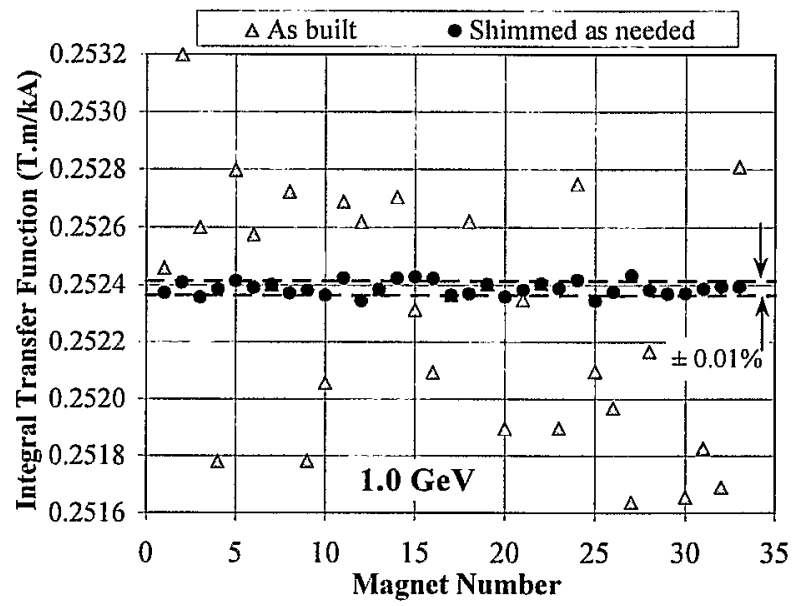

Figure 3: Integral dipole field at $1.0 \mathrm{GeV}$ before (triangles) and after (circles) shimming.

the nominal pole gap and the amount of correction required. As data on more shimmed magnets became available, an empirical relation between the shim thickness and the change in the ITF was established (see Fig. 2). This empirical relationship was used in the later part of the program for a more accurate shimming. A small difference was observed between the sensitivities for the return leg and pole shims. This difference is perhaps due to different levels of iron saturation in the pole and return leg regions.

Integral field measurements of the 32 magnets, at the central position, before and after shimming, are shown in Fig. 3. Before shimming, the rms variation of the integral field was $0.165 \%$; after shimming, it is $0.01 \%$. At $1.3 \mathrm{GeV}$, shimming reduced the rms variation from $0.089 \%$ to $0.033 \%$ (Fig. 4). Local dipole correctors will be used to correct the orbits. To minimize the load on these correctors, the dipoles will be sorted based on their integral field at $1.3 \mathrm{GeV}$. Magnets with high and low integral fields will be placed 360 degrees in phase advance (two full cells) apart.

With the measuring coil at the central position,

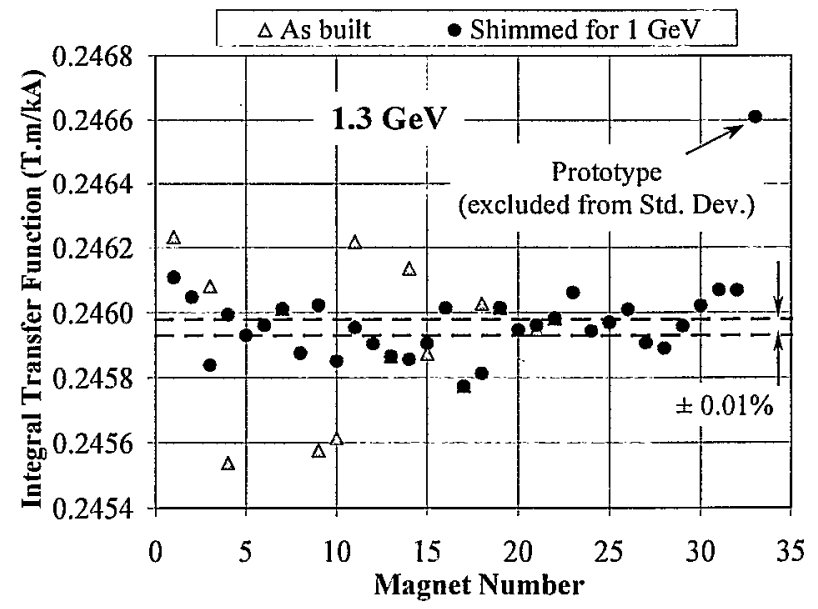

Figure 4: Integral dipole field at $1.3 \mathrm{GeV}$ before (triangles) and after (circles) shimming. 


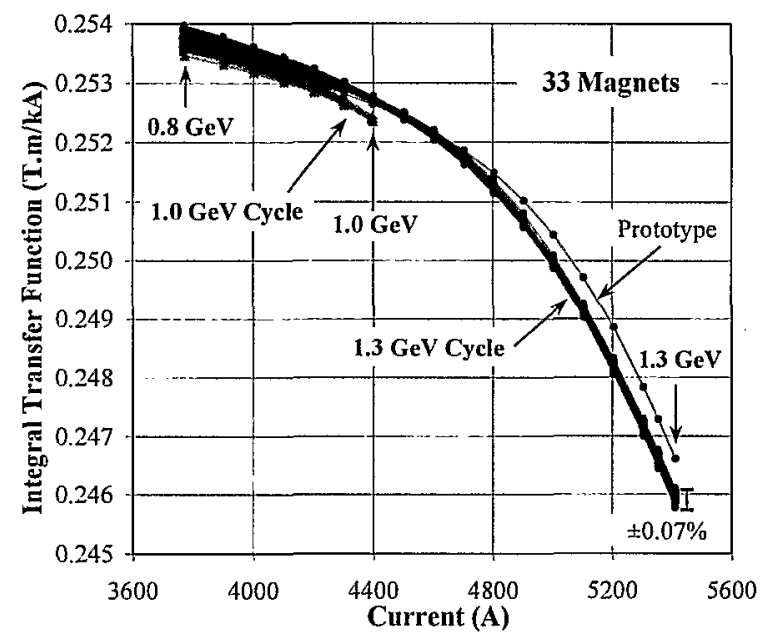

Figure 5: Excitation curves for operation at $1.0 \mathrm{GeV}$ and $1.3 \mathrm{GeV}$.

excitation curves for operation at 1.0 and $1.3 \mathrm{GeV}$ were measured, down to $0.8 \mathrm{GeV}$. Fig. 5 shows the excitation curves in the two cases.

An alternate way to represent the field quality of the magnets is to give the harmonic decomposition of the field. Table 2 (Table 3) summarizes the integral harmonics in the magnets at the central position of the

Table 2: Integral harmonics in the 17D120 dipoles in $10^{-4}$ units at $80 \mathrm{~mm}$ ( $1.0 \mathrm{GeV}$ operation)

\begin{tabular}{|c|c|c|c|c|}
\hline \multirow{2}{*}{ Harmonic } & \multicolumn{2}{|c|}{ Normal } & \multicolumn{2}{c|}{ Skew } \\
\cline { 2 - 5 } & Mean & Std. Dev. & Mean & Std. Dev. \\
\hline Quadrupole & -105.04 & 0.28 & -0.26 & 0.95 \\
\hline Sextupole & 0.16 & 0.43 & -0.02 & 0.49 \\
\hline Octupole & 2.05 & 0.15 & -0.05 & 0.32 \\
\hline Decapole & 1.14 & 0.20 & 0.00 & 0.11 \\
\hline Dodecapole & 0.06 & 0.09 & 0.00 & 0.16 \\
\hline 14-pole & -0.30 & 0.12 & -0.02 & 0.10 \\
\hline 16-pole & 0.15 & 0.07 & 0.00 & 0.10 \\
\hline 18-pole & -0.08 & 0.11 & 0.00 & 0.06 \\
\hline 20-pole & -0.04 & 0.05 & 0.01 & 0.09 \\
\hline 22-pole & -0.18 & 0.13 & 0.00 & 0.03 \\
\hline
\end{tabular}

Table 3: Integral harmonics in the $17 \mathrm{D} 120$ dipoles in $10^{-4}$ units at $80 \mathrm{~mm}$ ( $1.3 \mathrm{GeV}$ operation)

\begin{tabular}{|c|c|c|c|c|}
\hline \multirow{2}{*}{ Harmonic } & \multicolumn{2}{|c|}{ Normal } & \multicolumn{2}{c|}{ Skew } \\
\cline { 2 - 5 } & Mean & Std. Dev. & Mean & Std. Dev. \\
\hline Quadrupole & -103.66 & 0.29 & -0.19 & 1.01 \\
\hline Sextupole & -6.28 & 0.41 & -0.02 & 0.53 \\
\hline Octupole & 2.48 & 0.16 & -0.03 & 0.32 \\
\hline Decapole & -0.45 & 0.20 & 0.00 & 0.11 \\
\hline Dodecapole & 0.08 & 0.10 & 0.00 & 0.16 \\
\hline 14-pole & -0.49 & 0.12 & -0.02 & 0.10 \\
\hline 16-pole & 0.15 & 0.07 & 0.00 & 0.10 \\
\hline 18-pole & -0.07 & 0.11 & 0.00 & 0.06 \\
\hline 20-pole & -0.04 & 0.05 & 0.01 & 0.09 \\
\hline 22-pole & -0.17 & 0.13 & 0.00 & 0.03 \\
\hline
\end{tabular}

measuring coil at $1.0 \mathrm{GeV}(1.3 \mathrm{GeV})$ excitation. It is important to note that the integral measurements of two harmonics are affected by the magnet taper, which produces an apparent normal quadrupole of -105 units $(\sim 1 \%)$ and an apparent normal octupole of $\sim 2$ units. A three-dimensional calculation shows that the apparent values of these harmonics are -107 and +1.96 units respectively, in reasonable agreement with the measured values. The harmonics are essentially unchanged by the shimming. Both 2-D and 3-D calculations were performed to show that changes in harmonics due to the shims are negligible. Typical harmonic changes are less than 0.1 unit. The normal sextupole increases from 0 to 6 units between 1.0 and $1.3 \mathrm{GeV}$, the result of saturation. The $\sim 1$ unit variation of the skew quadrupole may be the result of top-bottom differences in the taper of the yoke.

The three-dimensional calculation also allows us to evaluate the difference between the harmonics measured by the (straight) integral coil and those along the curved path of a proton. Except for the normal quadrupole and normal octupole as discussed above, the harmonics integrated along the trajectory of the protons are expected to be within 1 unit of those measured by the rotating coil. The integral normal quadrupole and the normal octupole for the curved path differ from the straight path value by 99.4 units and 2.7 units respectively.

\section{CONCLUSIONS}

The 32 ring dipoles for the Spallation Neutron Source have been measured and found to meet the field quality tolerances. When shimmed, the magnets meet the $0.01 \%$ tolerance on the rms variation of integral dipole field at $1.0 \mathrm{GeV}$. The rms variation at $1.3 \mathrm{GeV}, 0.033 \%$, will be compensated by sorting. The integral fields are uniform across the good field region of $x= \pm 100 \mathrm{~mm}$ within $0.01 \%$, except for a residual quadrupole, estimated at $\sim 6$ units due to the magnet taper.

\section{ACKNOWLEDGEMENTS}

We acknowledge useful discussions with G. Danby, S. Henderson, J. Galambos, and T. Hunter of SNS.

\section{REFERENCES}

[1] N. Holtkamp, "Status of the SNS," PAC03 (These proceedings).

[2] J. Wei et al, "Low-loss design for the high-intensity accumulator ring of the Spallation Neutron Source," Phys. Rev. ST-AB 3, 080101 (2000).

[3] P. Wanderer et al., "The SNS Ring Dipole Magnetic field Quality," Proc. EPAC 2002, p. 1317.

[4] See, for example, A. Jain, "Harmonic Coils," in Proc. CERN Accelerator School on Measurement and Alignment of Accelerator and Detector Magnets, CERN 98-05, p. 175-218 (1998). 